Article

\title{
The Double Roman Domination Numbers of Generalized Petersen Graphs $P(n, 2)$
}

\author{
Huiqin Jiang ${ }^{1}, \mathrm{Pu} \mathrm{Wu}^{2}$, Zehui Shao ${ }^{2}$ (D), Yongsheng Rao ${ }^{2}$ and Jia-Bao Liu ${ }^{3, *}$ (iD \\ 1 Key Laboratory of Pattern Recognition and Intelligent Information Processing, Institutions of Higher \\ Education of Sichuan Province, Chengdu University, Chengdu 610106, China; hq.jiang@hotmail.com \\ 2 Institute of Computing Science and Technology, Guangzhou University, Guangzhou 510006, China; \\ puwu1997@126.com (P.W.); zshao@gzhu.edu.cn (Z.S.); rysheng@gzhu.edu.cn (Y.R.) \\ 3 School of Mathematics and Physics, Anhui Jianzhu University, Hefei 230601, China \\ * Correspondence: liujiabao@ahjzu.edu.cn or liujiabaoad@163.com
}

Received: 11 September 2018; Accepted: 10 October 2018; Published: 16 October 2018

\begin{abstract}
A double Roman dominating function (DRDF) $f$ on a given graph $G$ is a mapping from $V(G)$ to $\{0,1,2,3\}$ in such a way that a vertex $u$ for which $f(u)=0$ has at least a neighbor labeled 3 or two neighbors both labeled 2 and a vertex $u$ for which $f(u)=1$ has at least a neighbor labeled 2 or 3. The weight of a DRDF $f$ is the value $w(f)=\sum_{u \in V(G)} f(u)$. The minimum weight of a DRDF on a graph $G$ is called the double Roman domination number $\gamma_{d R}(G)$ of $G$. In this paper, we determine the exact value of the double Roman domination number of the generalized Petersen graphs $P(n, 2)$ by using a discharging approach.
\end{abstract}

Keywords: double Roman domination; discharging approach; generalized Petersen graphs

\section{Introduction}

In this paper, only graphs without multiple edges or loops are considered. For two vertices $u$ and $v$ of a graph $G$, we say $u \sim v$ in $G$ if $u v \in E(G)$. For positive integer $k$ and $u, v \in V(G)$, let $d(u, v)$ be the distance between $u$ and $v$ and $N_{k}(v)=\{u \mid d(u, v)=k\}$. The neighborhood of $v$ in $G$ is defined to be $N_{1}(v)$ (or simply $N(v)$ ). The closed neighborhood $N[v]$ of $v$ in $G$ is defined to be $N[v]=\{v\} \cup N(v)$. For a vertex subset $S \subseteq V(G)$, we denote by $G[S]$ the subgraph induced by $S$. For a positive integer $n$, we denote $[n]=\{1,2, \cdots, n\}$. For a set $S=\left\{x_{1}, x_{2}, \cdots, x_{n}\right\}$, if $x_{i}=x_{j}$ for some $i$ and $j$, then $S$ is considered as a multiset. Otherwise, $S$ is an ordinary set.

For positive integer numbers $n$ and $k$ with $n$ at least $2 k+1$, the generalized Petersen graph $P(n, k)$ is a graph with its vertex set $\left\{u_{i} \mid i=1,2, \cdots, n\right\} \cup\left\{v_{i} \mid i=1,2, \cdots, n\right\}$ and its edge set the union of $\left\{u_{i} u_{i+1}, u_{i} v_{i}, v_{i} v_{i+k}\right\}$ for $1 \leq i \leq n$, where subscripts are reduced modulo $n$ (see [1]).

A subset $D$ of the vertex set of a graph $G$ is a dominating set if every vertex in $V(G) \backslash D$ has at least one neighbor in $D$. The domination number, denoted by $\gamma(G)$, is the minimum number of vertices over all dominating sets of $G$.

There have been more than 200 papers studying various domination on graphs in the literature [2-6]. Among them, Roman domination and double Roman domination appear to be a new variety of interest [3,7-15].

A double Roman dominating function (DRDF) $f$ on a given graph $G$ is a mapping from $V(G)$ to $\{0,1,2,3\}$ in such a way that a vertex $u$ for which $f(u)=0$ has at least a neighbor labeled 3 or two neighbors both labeled 2 and a vertex $u$ for which $f(u)=1$ has at least a neighbor labeled 2 or 3 . The weight of a DRDF $f$ is the value $w(f)=\sum_{u \in V(G)} f(u)$. The minimum weight of a DRDF on a graph $G$ is called the double Roman domination number $\gamma_{d R}(G)$ of $G$. A DRDF $f$ of $G$ with $w(f)=\gamma_{d R}(G)$ 
is called a $\gamma_{d R}(G)$-function. Given a DRDF $f$ of $G$, we denote $E_{\left\{x_{1}, x_{2}\right\}}^{f}=\{u v \in E(G) \mid\{f(u), f(v)\}=$ $\left.\left\{x_{1}, x_{2}\right\}\right\}$. A graph $G$ is a double Roman Graph if $\gamma_{d R}(G)=3 \gamma(G)$.

In [7], Beeler et al. obtained the following results:

Proposition 1 ([7]). In a double Roman dominating function of weight $\gamma_{d R}(G)$, no vertex needs to be assigned the value one.

By Proposition 1, we now consider the DRDF of a graph $G$ in which there exists no vertex assigned with one in the following.

Given a DRDF $f$ of a graph $G$, suppose $\left(V_{0}^{f}, V_{2}^{f}, V_{3}^{f}\right)$ is the ordered partition of the vertex set of $G$ induced by $f$ in such a way that $V_{i}^{f}=\{v: f(v)=i\}$ for $i=0,2,3$. It can be seen that there is a 1-1 mapping between $f$ and $\left(V_{0}^{f}, V_{2}^{f}, V_{3}^{f}\right)$, and we write $f=\left(V_{0}^{f}, V_{2}^{f}, V_{3}^{f}\right)$, or simply $\left(V_{0}, V_{2}, V_{3}\right)$. Given a DRDF $f$ of $P(n, 2)$ and letting $w_{i} \in\{0,2,3\}$ for $i=1,2,3$ with $w_{1} \geq w_{2} \geq w_{3}$, we write $V_{j}^{w_{1} w_{2} w_{3}}=$ $\left\{x \in V(P(n, 2)) \mid f(x)=j,\left\{w_{1}, w_{2}, w_{3}\right\}=\left\{f\left(x_{1}\right), f\left(x_{2}\right), f\left(x_{3}\right)\right\}\right\}$, where $N(x)=\left\{x_{1}, x_{2}, x_{3}\right\}$.

Now, we will use $f(\cdot)=q^{+}$to represent the value scope $f(\cdot) \geq q$ for an integer $q$. We say a path $t_{1} t_{2} \cdots t_{k}$ is a path of type $c_{1}-c_{2}-\cdots-c_{k}$ if $f\left(t_{i}\right)=c_{i}$ for $i \in[k]$. Let $H$ be a subgraph induced by five vertices $s_{1}, s_{2}, s_{3}, s_{4}, s_{5}$ with $s_{1} \sim s_{2}, s_{2} \sim s_{3}, s_{3} \sim s_{4}, s_{3} \sim s_{5}$ satisfying $f\left(s_{3}\right)=0$ and $f\left(s_{1}\right)=a$, $f\left(s_{2}\right)=b, f\left(s_{4}\right)=c, f\left(s_{5}\right)=d$ for some $a, b, c, d \in\{0,2,3\}$, then we say $H$ is a subgraph of type $a-b-0_{-d}^{-c}$.

Let $W$ be a subgraph induced by four vertices $s_{1}, s_{2}, s_{3}, s_{4}$ with $s_{1} \sim s_{2}, s_{2} \sim s_{3}, s_{2} \sim s_{4}$, satisfying $f\left(s_{1}\right)=a, f\left(s_{2}\right)=0, f\left(s_{3}\right)=b$ and $f\left(s_{4}\right)=c$ for some $a, b, c \in\{0,2,3\}$, then we say $W$ is a subgraph of type $a-0_{-c}^{-b}$.

In the graph $P(n, 2)$, we will denote the set of vertices of $\left\{u_{i}, v_{i}\right\}$ with $L^{(i)}$. For a given DRDF $f$ of $P(n, 2)$, let $w_{f}\left(L^{(i)}\right)$ denote the weight of $L^{(i)}$, that is $w_{f}\left(L^{(i)}\right)=\sum_{u \in V\left(L^{(i)}\right)} f(u)$. Let $\mathcal{B}_{i}=\left\{L^{(i-2)}, L^{(i-1)}\right.$, $\left.L^{(i)}, L^{(i+1)}, L^{(i+2)}\right\}$, where the subscripts are taken modulo $n$. We define $w_{f}\left(\mathcal{B}_{i}\right)=\sum_{j=-2}^{2} w_{f}\left(L^{(i+j)}\right)$, and:

$$
f\left(\mathcal{B}_{i}\right)=f\left(\begin{array}{ccccc}
u_{i-2} & u_{i-1} & u_{i} & u_{i+1} & u_{i+2} \\
v_{i-2} & v_{i-1} & v_{i} & v_{i+1} & v_{i+2}
\end{array}\right) .
$$

Motivation: Beeler et al. [7] put forward an open problem about characterizing the double Roman graphs. As an interesting family of graphs, the domination and its variations of generalized Petersen graphs have attracted considerable attention $[1,16]$. Therefore, it is interesting to characterize the double Roman graphs in generalized Petersen graphs. In this paper, we focus on finding the double Roman graphs in $P(n, 2)$.

\section{Double Roman Domination Number of $P(n, 2)$}

2.1. Upper Bound for the Double Roman Domination Number of $P(n, 2)$

Lemma 1. If $n \geq 5$, then:

$$
\gamma_{d R}(P(n, 2)) \leq \begin{cases}\left\lceil\frac{8 n}{5}\right\rceil, & n \equiv 0(\bmod 5) \\ \left\lceil\frac{8 n}{5}\right\rceil+1, & n \equiv 1,2,3,4(\bmod 5)\end{cases}
$$

Proof. We consider the following five cases.

Case $1: n \equiv 0(\bmod 5)$.

Let:

$$
P_{5}=\left[\begin{array}{lllll}
2 & 0 & 2 & 0 & 0 \\
0 & 0 & 0 & 2 & 2
\end{array}\right]
$$


Then, by repeating the pattern of $P_{5}$, we obtain a DRDF of weight $8 k$ of $P(5 k, 2)$, and the upper bound is obtained.

Case $2: n \equiv 1(\bmod 5)$.

If $n=6$, let:

$$
P_{6}=\left[\begin{array}{llllll}
0 & 2 & 0 & 2 & 0 & 0 \\
2 & 0 & 0 & 0 & 2 & 3
\end{array}\right] .
$$

Then, the pattern $P_{6}$ induces a DRDF of weight 11 of $P(6,2)$, and the desired upper bound is obtained.

If $n \geq 11$, let:

$$
P_{11}=\left[\begin{array}{lllllllllll}
2 & 0 & 2 & 0 & 0 & 2 & 2 & 0 & 2 & 0 & 0 \\
0 & 0 & 0 & 2 & 2 & 0 & 0 & 0 & 0 & 3 & 2
\end{array}\right] .
$$

Then, by repeating the leftmost five columns of the pattern of $P_{11}$, we obtain a DRDF of weight $8 k+3$ of $P(5 k+1,2)$, and the desired upper bound is obtained.

Case $3: n \equiv 2(\bmod 5)$.

If $n=7$, let:

$$
P_{7}=\left[\begin{array}{lllllll}
2 & 0 & 2 & 0 & 0 & 3 & 0 \\
0 & 0 & 2 & 2 & 0 & 0 & 2
\end{array}\right] .
$$

Then, the pattern $P_{7}$ induces a DRDF of weight 13 of $P(7,2)$, and the desired upper bound is obtained.

If $n \geq 12$, let:

$$
P_{12}=\left[\begin{array}{llllllllllll}
2 & 0 & 2 & 0 & 0 & 2 & 0 & 3 & 0 & 2 & 0 & 0 \\
0 & 0 & 0 & 2 & 2 & 0 & 2 & 0 & 0 & 0 & 2 & 2
\end{array}\right] .
$$

Then, by repeating the leftmost five columns of the pattern of $P_{12}$, we obtain a DRDF of weight $8 k+6$ of $P(5 k+2,2)$, and the desired upper bound is obtained.

Case $4: n \equiv 3(\bmod 5)$.

If $n \geq 8$, let:

$$
P_{8}=\left[\begin{array}{llllllll}
2 & 0 & 2 & 0 & 0 & 2 & 0 & 0 \\
0 & 0 & 0 & 2 & 2 & 0 & 2 & 2
\end{array}\right] .
$$

Then, by repeating the leftmost five columns of the pattern of $P_{8}$, we obtain a DRDF of weight $8 k+6$ of $P(5 k+3,2)$, and the desired upper bound is obtained.

Case $5: n \equiv 4(\bmod 5)$.

If $n \geq 9$, let:

$$
P_{9}=\left[\begin{array}{ccccccccc}
2 & 0 & 2 & 0 & 0 & 3 & 0 & 0 & 0 \\
0 & 0 & 0 & 2 & 2 & 0 & 0 & 3 & 2
\end{array}\right] .
$$

Then, by repeating the leftmost five columns of the pattern of $P_{9}$, we obtain a DRDF of weight $8 k+8$ of $P(5 k+4,2)$, and the desired upper bound is obtained.

\subsection{Lower Bound for Double Roman Domination Number of $P(n, 2)$}

Lemma 2. Let $f$ be a $\gamma_{d R}$-function of $P(n, 2)$ with $n \geq 5$. Then, $w_{f}\left(\mathcal{B}_{i}\right) \geq 4$.

Proof. Since $u_{i}, v_{i}, u_{i+1}$ and $u_{i-1}$ need to be double Roman dominated by vertices in $\mathcal{B}_{i}$, we have $w_{f}\left(\mathcal{B}_{i}\right) \geq 3$. Now, we will show that $w_{f}\left(\mathcal{B}_{i}\right) \neq 3$. Otherwise, it is clear that $f\left(u_{i}\right)=3$, and $f(x)=0$ for any $x \in \mathcal{B}_{i} \backslash\left\{u_{i}\right\}$. Since $v_{i \pm 1}, u_{i \pm 2}$ and $v_{i \pm 2}$ need to be double Roman dominated, we have 
$f\left(u_{i \pm 3}\right)=f\left(v_{i \pm 3}\right)=f\left(v_{i \pm 4}\right)=3$. Now, we can obtain a DRDF $f^{\prime}$ from $f$ by letting $f^{\prime}\left(u_{i-2}\right)=2$, $f^{\prime}\left(u_{i-3}\right)=0$ and $f^{\prime}(v)=f(v)$ for $v \in V(P(n, 2)) \backslash\left\{u_{i-2}, u_{i-3}\right\}$. Then, we have $w\left(f^{\prime}\right)<w(f)$, a contradiction (see Figure 1). Therefore, $w_{f}\left(\mathcal{B}_{i}\right) \geq 4$.

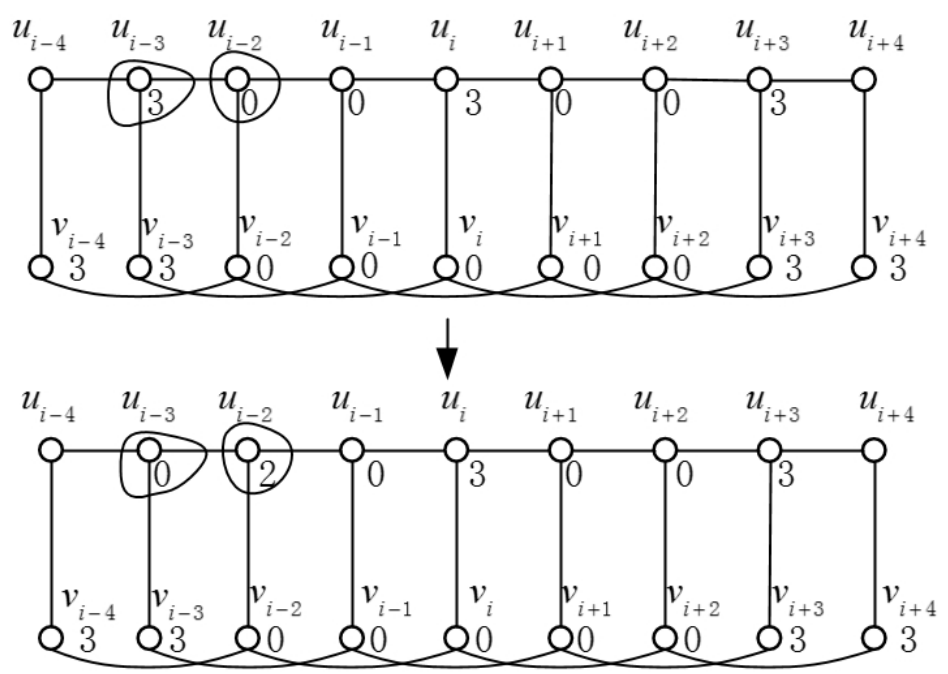

Figure 1. Construct a function $f^{\prime}$ from $f$ used in Lemma 2.

Lemma 3. Let $f$ be a $\gamma_{d R}$-function of $P(n, 2)$ with $n \geq 5$. Then, for any $i \in[n]$, it is impossible that $f\left(v_{i-1}\right)=f\left(v_{i}\right)=f\left(v_{i+1}\right)=3$ and $f(x)=0$ for any $x \in \mathcal{B}_{i} \backslash\left\{v_{i-1}, v_{i}, v_{i+1}\right\}$.

Proof. Suppose to the contrary that $f\left(v_{i-1}\right)=f\left(v_{i}\right)=f\left(v_{i+1}\right)=3$ and $f(x)=0$ for $x \in \mathcal{B}_{i} \backslash$ $\left\{v_{i-1}, v_{i}, v_{i+1}\right\}$. Then, we have $f\left(u_{i \pm 3}\right)=3$. Now, we can obtain a DRDF $f^{\prime}$ from $f$ by letting $f^{\prime}\left(u_{i-1}\right)=2, f^{\prime}\left(v_{i-1}\right)=0$ and $f^{\prime}(v)=f(v)$ for $v \in V(P(n, 2)) \backslash\left\{v_{i-1}, u_{i-1}\right\}$. Then, we have $w\left(f^{\prime}\right)<w(f)$, a contradiction (see Figure 2).

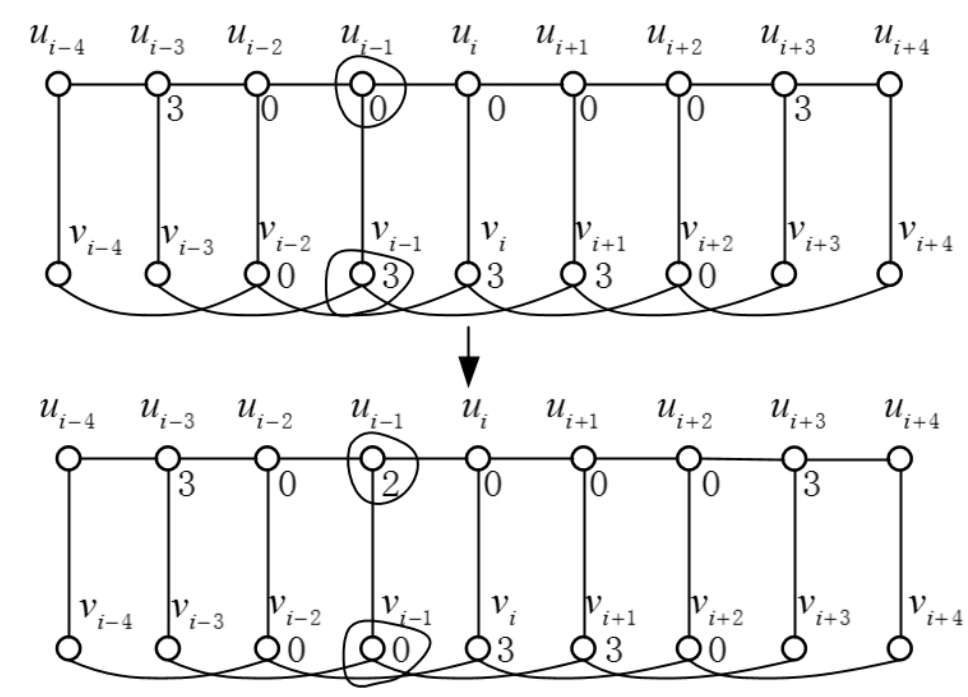

Figure 2. Construct a function $f^{\prime}$ from $f$ in Lemma 3.

Lemma 4. Let $f$ be a $\gamma_{d R}$-function of $P(n, 2)$ with $n \geq 5$. Then, for each $x \in V_{3}^{000}$, there exists a neighbor $y$ of $x$ such that $y \in V_{0}^{320} \cup V_{0}^{330} \cup V_{0}^{322} \cup V_{0}^{332} \cup V_{0}^{333}$, or equivalently, it is impossible that for any $x \in V_{3}^{000}$, $f(z)=0$ for any $z \in N_{2}(x)$. 
Proof. Suppose to the contrary that there is a vertex $x \in V_{3}^{000}$ such that $y \in V_{0}^{300}$ for every neighbor $y$ of $x$. Now, it is sufficient to consider the following two cases.

Case 1: $x=u_{i}$ for some $i$.

In this case, we have $f\left(u_{i}\right)=3$ and $f(x)=0$ for $x \in \mathcal{B}_{i} \backslash\left\{u_{i}\right\}$. Then, we have $w_{f}\left(\mathcal{B}_{i}\right)=3<4$, contradicting Lemma 2.

Case 2: $x=v_{i}$ for some $i$.

In this case, since $u_{i \pm 1}$ and $u_{i \pm 2}$ need to be double Roman dominated, we have $f\left(v_{i \pm 1}\right)=3$ and $f\left(u_{i \pm 3}\right)=3$. By Lemma 3 , such a case is impossible.

Discharging procedure: Let $f$ be a DRDF of $P(n, 2)$. We set the initial charge of every vertex $x$ be $s(x)=f(x)$. We use the discharging procedure, leading to a final charge $s^{\prime}$, defined by applying the following rules:

R1: $\quad$ Each $s(x)$ for which $s(x)=3$ transmits 0.8 charge to each neighbor $y$ with $y \in V_{0}^{300}$ transmits 0.6 charge to each neighbor $y$ with $y \in V_{0}^{320} \cup V_{0}^{330} \cup V_{0}^{322} \cup V_{0}^{332} \cup V_{0}^{333}$.

R2: $\quad$ Each $s(x)$ for which $s(x)=2$ transmits 0.4 charge to each neighbor $y$ with $y \in V_{0}$.

Proposition 2. If $n \geq 5$, then $\gamma_{d R}(P(n, 2)) \geq\left\lceil\frac{8 n}{5}\right\rceil$.

Proof. Assume $f$ is a $\gamma_{d R}$-function of $P(n, 2)$. We use the above discharging procedure. Now, it is sufficient to consider the following three cases.

Case 1: By Lemma 4, there exists a vertex $z$ with $f(z) \geq 2$ for some $z \in N_{2}(x)$, for any $x \in V_{3}^{000}$. Therefore, by rule $\mathrm{R} 1$, for each $v \in V_{3}^{000}$, the final charge $s^{\prime}(v)$ is at least $3-0.6-0.8-0.8=0.8$. For each $v \in V_{3} \backslash V_{3}^{000}$, then the final charge $s^{\prime}(v)$ is at least $3-0.8-0.8=1.4$.

Case 2: By rule $\mathrm{R} 2$, for each $v \in V_{2}$, the final charge $s^{\prime}(v)$ is at least $2-0.4-0.4-0.4=0.8$.

Case 3: For each $v \in V_{0}^{300}$, the final charge $s^{\prime}(v)$ is 0.8 by rule R1. For each $v \in V_{0} \backslash V_{0}^{300}$, the final charge $s^{\prime}(v)$ is at least 0.8 by rules $\mathrm{R} 1$ and $\mathrm{R} 2$.

From the above, we have:

$$
s^{\prime}(v) \geq 0.8 \text { for any } v \in P(n, 2)
$$

Hence, $w(f)=\sum_{v \in V(P(n, 2))} s(v)=\sum_{v \in V(P(n, 2))} s^{\prime}(v) \geq 0.8 \times 2 n=\frac{8 n}{5}$. Since $w(f)$ is an integer, we have $w(f) \geq\left\lceil\frac{8 n}{5}\right\rceil$.

By using the above discharging rules, we have the following lemma immediately, and the proof is omitted.

Lemma 5. Let $f$ be a $\gamma_{d R}$-function of $P(n, 2)$ with $n \geq 5$. If we use the above discharging procedure for $f$ on $P(n, 2)$, then:

(a) if there exists a path $P$ of type $2-2-2$, or type $2^{+}-3$, or type $2-2-0-3$, or type $3-0-2^{+}-$ $0-3-0-2^{+}-0-3$, or type $3-0-2^{+}-0-3-0-3$, or type $3-0-3-0-3$, or type $2^{+}-0-3-0-3-0-2^{+}$or a subgraph $P$ of type $3-0_{-3}^{-3}$, then $\sum_{v \in V(P)}\left(s^{\prime}(v)-0.8\right) \geq 1$.

(b) if there exist a path $P_{1}$ of type $2-2$ and a path $P_{2}$ of type $2^{+}-0-3$, then $\sum_{v \in V\left(P_{1}\right) \cup V\left(P_{2}\right)}\left(s^{\prime}(v)-0.8\right) \geq$ 1.

(c) if there exists a subgraph $H$ of type $2-2-0_{-2}^{-2}$, then $\sum_{v \in V(H)}\left(s^{\prime}(v)-0.8\right) \geq 1.2$.

(d) if there exist a path $P$ of type $3-0-3$, together with a subgraph $H$ of type $2^{+}-0-3-0-2^{+}$or type $3-0_{-2^{+}}^{-2^{+}}$, then $\sum_{v \in V(P) \cup V(H)}\left(s^{\prime}(v)-0.8\right) \geq 1$.

(e) if there exist three paths $P_{1}, P_{2}, P_{3}$ of type $3-0-3$, then $\sum_{v \in V\left(P_{1}\right) \cup V\left(P_{2}\right) \cup V\left(P_{3}\right)}\left(s^{\prime}(v)-0.8\right) \geq 1.2$. 
Lemma 6. Let $f$ be a $\gamma_{d R}$-function of $P(n, 2)$ with weight $\left\lceil\frac{8 n}{5}\right\rceil$, then there exists no edge uv $\in E(P(n, 2))$ for which $u v \in E_{\{2,2\}}^{f} \cup E_{\{2,3\}}^{f} \cup E_{\{3,3\}}^{f}$.

Proof. First, we have:

$$
\gamma_{d R}(P(n, 2))=w(f)=\left\lceil\frac{8 n}{5}\right\rceil \leq \frac{8 n+4}{5}=\frac{8 n}{5}+0.8
$$

and so:

$$
w(f)-\frac{8 n}{5} \leq 0.8
$$

We use the above discharging procedure for $f$ on $P(n, 2)$, and similar to the proof of Proposition 2, we have:

$$
w(f)=\sum_{v \in V(P(n, 2))} s^{\prime}(v)
$$

and so:

$$
\sum_{v \in V(P(n, 2))}\left(s^{\prime}(v)-\frac{4}{5}\right) \leq 0.8
$$

By Lemma 5a and Equation (2), we have that there exists no edge $u v \in E_{\{2,3\}}^{f} \cup E_{\{3,3\}}^{f}$.

Now, suppose to the contrary that there exists an edge $u v \in E_{\{2,2\}}^{f}$, and it is sufficient to consider the following three cases.

Case 1: $f\left(u_{i}\right)=f\left(u_{i+1}\right)=2$.

We have $f\left(u_{i-1}\right)=f\left(u_{i+2}\right)=f\left(v_{i+1}\right)=f\left(v_{i}\right)=0$. Otherwise, there exists a path $P$ of type $2-2-2$ or type $2^{+}-3$. By Lemma 5 a, we have $\sum_{v \in V(P)}\left(s^{\prime}(v)-0.8\right) \geq 1$, contradicting Equation (2).

Since $u_{i+2}$ needs to be double Roman dominated, we have $\left\{f\left(u_{i+3}\right), f\left(v_{i+2}\right)\right\}=\{0,2\}$. Otherwise, $f(x)=3$ for some $x \in\left\{u_{i+3}, v_{i+2}\right\}$ or $f\left(u_{i+3}\right)=f\left(v_{i+2}\right)=2$.

If $f(x)=3$ for some $x \in\left\{u_{i+3}, v_{i+2}\right\}$, there exists a path $P$ of type $2-2-0-3$. By Lemma 5a, we have $\sum_{v \in V(P)}\left(s^{\prime}(v)-0.8\right) \geq 1$, contradicting Equation (2).

If $f\left(u_{i+3}\right)=f\left(v_{i+2}\right)=2$, there exists a subgraph $H$ of type $2-2-0_{-2}^{-2}$. By Lemma $5 c$, we have $\sum_{v \in V(H)}\left(s^{\prime}(v)-0.8\right) \geq 1.2$, contradicting Equation (2).

Now, it is sufficient to consider the following two cases.

Case 1.1: $f\left(v_{i+2}\right)=2, f\left(u_{i+3}\right)=0$.

To double Roman dominate $v_{i+1}$, we have $f\left(v_{i+3}\right) \geq 2$ or $f\left(v_{i-1}\right) \geq 2$. First, we have $f\left(v_{i+3}\right) \neq 3$ and $f\left(v_{i-1}\right) \neq 3$. Otherwise, $u_{i} u_{i+1} v_{i+1} v_{i+3}$ or $u_{i} u_{i+1} v_{i+1} v_{i-1}$ is a path $P$ of type $2-2-0-3$. By Lemma 5a, we have $\sum_{v \in V(P)}\left(s^{\prime}(v)-0.8\right) \geq 1$, contradicting Equation (2).

Now, we have that it is impossible $f\left(v_{i+3}\right)=f\left(v_{i-1}\right)=2$. Otherwise, the set $\left\{u_{i}, u_{i+1}, v_{i+1}, v_{i+3}, v_{i-1}\right\}$ induces a subgraph $H$ of type $2-2-0_{-2}^{-2}$. By Lemma $5 \mathrm{c}$, we have $\sum_{v \in V(H)}\left(s^{\prime}(v)-0.8\right) \geq 1.2$, contradicting Equation (2).

Therefore, we have $\left\{f\left(v_{i+3}\right), f\left(v_{i-1}\right)\right\}=\{0,2\}$. Now, it is sufficient to consider the following two cases.

Case 1.1.1: $f\left(v_{i+3}\right)=2, f\left(v_{i-1}\right)=0$.

Since $v_{i-1}$ and $u_{i-1}$ need to be double Roman dominated, we have $f\left(v_{i-3}\right)=3, f\left(u_{i-2}\right)=2^{+}$. Then, there exists a path $P_{1}$ of type $2-2$ and a path $P_{2}$ of type $2^{+}-0-3$. By Lemma $5 \mathrm{~b}$, we have $\sum_{v \in V\left(P_{1}\right) \cup V\left(P_{2}\right)}\left(s^{\prime}(v)-0.8\right) \geq 1$, contradicting Equation (2).

Case 1.1.2: $f\left(v_{i+3}\right)=0, f\left(v_{i-1}\right)=2$. 
Since $u_{i+3}$ and $v_{i+3}$ need to be double Roman dominated, we have $f\left(u_{i+4}\right)=f\left(v_{i+5}\right)=3$. Then, there exist a path $P_{1}$ of type $2-2$ and a path $P_{2}$ of type $3-0-3$. By Lemma $5 \mathrm{~b}$, $\sum_{v \in V\left(P_{1}\right) \cup V\left(P_{2}\right)}\left(s^{\prime}(v)-0.8\right) \geq 1$, contradicting Equation (2).

Case 1.2: $f\left(v_{i+2}\right)=0, f\left(u_{i+3}\right)=2$.

Since $v_{i+2}$ needs to be double Roman dominated, we have $f\left(v_{i+4}\right)=3$. Then, there exist a path $P_{1}$ of type $2-2$ and a path $P_{2}$ of type $2-0-3$. By Lemma $5 \mathrm{~b}, \sum_{v \in V\left(P_{1}\right) \cup V\left(P_{2}\right)}\left(s^{\prime}(v)-0.8\right) \geq 1$, contradicting Equation (2).

Case 2: $f\left(v_{i}\right)=f\left(u_{i}\right)=2$.

We have $f\left(u_{i \pm 1}\right)=f\left(v_{i \pm 2}\right)=0$. Otherwise, there exists a path $P$ of type $2-2-2$ or type $2^{+}-3$. By Lemma 5 a, we have $\sum_{v \in V(P)}\left(s^{\prime}(v)-0.8\right) \geq 1$, contradicting Equation (2).

Since $u_{i+1}$ needs to be double Roman dominated, we have $\left\{f\left(u_{i+2}\right), f\left(v_{i+1}\right)\right\}=\{0,2\}$. Otherwise, by Lemma 5 a or Lemma $5 c$, we obtain a contradiction with Equation (2).

Now, we consider the following two subcases.

Case 2.1: $f\left(v_{i+1}\right)=2, f\left(u_{i+2}\right)=0$.

Since $u_{i+2}$ needs to be double Roman dominated, we have $f\left(u_{i+3}\right)=3$. Then, there exist a path $P_{1}$ of type $2-2$ and a path $P_{2}$ of type $2-0-3$. By Lemma $5 \mathrm{~b}, \sum_{v \in V\left(P_{1}\right) \cup V\left(P_{2}\right)}\left(s^{\prime}(v)-0.8\right) \geq 1$, contradicting Equation (2).

Case 2.2: $f\left(v_{i+1}\right)=0, f\left(u_{i+2}\right)=2$.

Since $v_{i+1}$ needs to be double Roman dominated, we have $f(x)=3$ for some $x \in\left\{v_{i+3}, v_{i-1}\right\}$ or $f\left(v_{i+3}\right)=f\left(v_{i-1}\right)=2$. If $f(x)=3$ for some $x \in\left\{v_{i+3}, v_{i-1}\right\}$, there exist a path $P_{1}$ of type $2-2$ and a path $P_{2}$ of type $2-0-3$. By Lemma $5 \mathrm{~b}, \sum_{v \in V\left(P_{1}\right) \cup V\left(P_{2}\right)}\left(s^{\prime}(v)-0.8\right) \geq 1$, contradicting Equation (2).

If $f\left(v_{i+3}\right)=f\left(v_{i-1}\right)=2$, then by Lemma $5 \mathrm{~b}$,c, we have $u_{i-2}=0$. Since $u_{i-2}$ needs to be double Roman dominated, we have $f\left(u_{i-3}\right)=3$. Then, there exist a path $P_{1}$ of type $2-2$ and a path $P_{2}$ of type $2-0-3$. By Lemma $5 \mathrm{~b}, \sum_{v \in V\left(P_{1}\right) \cup V\left(P_{2}\right)}\left(s^{\prime}(v)-0.8\right) \geq 1$, contradicting Equation (2).

Case 3: $f\left(v_{i+1}\right)=f\left(v_{i-1}\right)=2$.

We have $f\left(u_{i \pm 1}\right)=f\left(v_{i \pm 3}\right)=0$. Otherwise, there exists a path $P$ of type $2-2-2$ or type $2^{+}-3$. By Lemma $5 \mathrm{a}$, we have $\sum_{v \in V(P)}\left(s^{\prime}(v)-0.8\right) \geq 1$, contradicting Equation (2).

Since $u_{i}$ needs to be double Roman dominated, we have $f\left(u_{i}\right)=2$ or $f\left(v_{i}\right)=3$.

Case 3.1: $f\left(u_{i}\right)=2, f\left(v_{i}\right)=0$.

By Lemma $5 \mathrm{~b}, \mathrm{c}$ and Equation (2), we have $f\left(u_{i \pm 2}\right)=0$. Since $v_{i}$ needs to be double Roman dominated, we have $\left\{f\left(v_{i-2}\right), f\left(v_{i+2}\right)\right\}=\{0,2\}$. Considering isomorphism, we without loss of generality assume $f\left(v_{i+2}\right)=2$ and $f\left(v_{i-2}\right)=0$. Since $u_{i-2}$ needs to be double Roman dominated, $f\left(u_{i-3}\right)=3$. Then, there exist a path $P_{1}$ of type $2-2$ and a path $P_{2}$ of type $2-0-3$. By Lemma $5 \mathrm{~b}$, $\sum_{v \in V\left(P_{1}\right) \cup V\left(P_{2}\right)}\left(s^{\prime}(v)-0.8\right) \geq 1$, contradicting Equation (2).

Case 3.2: $f\left(u_{i}\right)=0, f\left(v_{i}\right)=3$.

By Lemma $5 \mathrm{a}$ and Equation (2), we have $f\left(v_{i \pm 2}\right)=0$. Since $u_{i+1}$ needs to be double Roman dominated, we have $f\left(u_{i+2}\right)=2$. Then, there exist a path $P_{1}$ of type $2-2$ and a path $P_{2}$ of type $2-0$ - 3. By Lemma $5 \mathrm{~b}, \sum_{v \in V\left(P_{1}\right) \cup V\left(P_{2}\right)}\left(s^{\prime}(v)-0.8\right) \geq 1$, contradicting Equation (2).

Therefore, the proof is complete.

Lemma 7. Let $f$ be a $\gamma_{d R}$-function of $P(n, 2)$ with weight $\left\lceil\frac{8 n}{5}\right\rceil, v \in V_{3}^{000}$ and $S=\left\{x \mid x \in N_{2}(v), f(x) \geq 2\right\}$, then $1 \leq|S| \leq 2$. 
Proof. We use the above discharging procedure for $f$ on $P(n, 2)$. By Lemma 4 , we have $|S| \geq 1$. Now, suppose to the contrary that $|S| \geq 3$. By rules R1 and R2 and Equation (1), we have:

$$
\sum_{v \in V(P(n, 2))}\left(s^{\prime}(v)-\frac{4}{5}\right) \geq \sum_{x \in N[v] \cup N_{2}(v)}\left(s^{\prime}(x)-\frac{4}{5}\right) \geq 1
$$

contradicting Equation (2).

Lemma 8. If $n \geq 5$ and $f$ is a $\gamma_{d R}$-function of $P(n, 2)$ with $f\left(u_{i}\right)=3$ for some $i \in[n]$, then $w(f) \geq\left\lceil\frac{8 n}{5}\right\rceil+1$.

Proof. Suppose to the contrary that there exists a $\gamma_{d R}$-function $f$ with $w(f)=\left\lceil\frac{8 n}{5}\right\rceil$ such that $f\left(u_{i}\right)=3$ for some $i \in[n]$. By Lemma 6, we have $f\left(v_{i}\right)=f\left(u_{i \pm 1}\right)=0$. Let $S=\left\{x \mid x \in N_{2}(v), f(x) \geq 2\right\}$. By Lemma 7, we have $|S| \in\{1,2\}$. Therefore, we just need to consider the following two cases.

Case 1: $|S|=1$.

We may w.l.o.g assume that $\left\{f\left(u_{i-2}\right), f\left(v_{i-1}\right), f\left(v_{i-2}\right)\right\}=\{0,0,2\}$ or $\{0,0,3\}$ and $f\left(v_{i+1}\right)=$ $f\left(v_{i+2}\right)=f\left(u_{i+2}\right)=0$. Since $u_{i+2}, v_{i+2}$ need to be double Roman dominated, we have $f\left(u_{i+3}\right)=$ $f\left(v_{i+4}\right)=3$, and thus, $f\left(v_{i+3}\right)=0$. Since $v_{i+1}$ needs to be double Roman dominated, we have $f\left(v_{i-1}\right)=3$. Thus, $f\left(u_{i-2}\right)=f\left(v_{i-2}\right)=0$. Since $u_{i-2}, v_{i-2}$ need to be double Roman dominated, we have $f\left(u_{i-3}\right)=f\left(v_{i-4}\right)=3$. Then, there exist three paths $P_{1}, P_{2}, P_{3}$ of type $3-0-3$. By Lemma $5 \mathrm{e}$, we have $\sum_{v \in V\left(P_{1}\right) \cup V\left(P_{2}\right) \cup V\left(P_{3}\right)}\left(s^{\prime}(v)-0.8\right) \geq 1.2$, contradicting Equation (2).

Case 2: $|S|=2$.

It is sufficient to consider the following cases.

Case 2.1: $S \subseteq\left\{v_{i-1}, v_{i-2}, u_{i-2}\right\}$ and $f\left(v_{i+1}\right)=f\left(v_{i+2}\right)=f\left(u_{i+2}\right)=0$.

Since $u_{i+2}, v_{i+2}$ need to be double Roman dominated, we have $f\left(u_{i+3}\right)=f\left(v_{i+4}\right)=3$. Then, there exist a path $P$ of type $3-0-3$, and a subgraph $H$ of type $2^{+}-0-3-0-2^{+}$or type $3-0_{-2^{+}}^{-2^{+}}$. By Lemma $5 \mathrm{~d}$, we have $\sum_{v \in V(P) \cup V(H)}\left(s^{\prime}(v)-0.8\right) \geq 1$, contradicting Equation (2).

Case 2.2: $S=\left\{s_{1}, s_{2}\right\}, s_{1} \in\left\{v_{i-1}, v_{i-2}, u_{i-2}\right\}$ and $s_{2} \in\left\{v_{i+1}, v_{i+2}, u_{i+2}\right\}$.

First, we have $f\left(v_{i \pm 1}\right)=0$. Otherwise, we may without loss of generality assume that $f\left(v_{i+1}\right) \geq 2$. Since $u_{i+2}, v_{i+2}$ need to be double Roman dominated, we have $f\left(u_{i+3}\right)=f\left(v_{i+4}\right)=3$. Then, there exist a path $P$ of type $3-0-3$, and a path $H$ of type $2^{+}-0-3-0-2^{+}$. By Lemma $5 \mathrm{~d}$, we have $\sum_{v \in V(P) \cup V(H)}\left(s^{\prime}(v)-0.8\right) \geq 1$, contradicting Equation (2).

Then, since $v_{i+1}, v_{i-1}$ need to be double Roman dominated, we have $f\left(v_{i+3}\right)=f\left(v_{i-3}\right)=3$. By Lemma 6, we have $f\left(u_{i+3}\right)=f\left(u_{i-3}\right)=0$. Since $u_{i \pm 2}$ need to be double Roman dominated, we have $\left(f\left(u_{i-2}\right), f\left(v_{i-2}\right)\right) \in\{(0,3),(2,0),(3,0)\}$ and $\left(f\left(u_{i+2}\right), f\left(v_{i+2}\right)\right) \in\{(0,3),(2,0),(3,0)\}$.

It is impossible that $f\left(v_{i+2}\right)+f\left(u_{i+2}\right)=3$ and $f\left(v_{i-2}\right)+f\left(u_{i-2}\right)=3$. Otherwise, there exists a path $P$ of type $3-0-3-0-3$ or a subgraph $P$ of type $3-0_{-3}^{-3}$. By Lemma 5 a, we have $\sum_{v \in V(P)}\left(s^{\prime}(v)-\right.$ $0.8) \geq 1$, contradicting Equation (2).

It is impossible $f\left(u_{i \pm 2}\right) \geq 2$. Otherwise, there exists a path $P$ of type $3-0-2^{+}-0-3-0-$ $2^{+}-0-3$. By Lemma $5 \mathrm{a}$, we have $\sum_{v \in V(P)}\left(s^{\prime}(v)-0.8\right) \geq 1$, contradicting Equation (2).

Then, we may without loss of generality assume that $f\left(u_{i+2}\right)=2$ and $f\left(v_{i-2}\right)=3$. Then, there exists a path $P$ of type $3-0-2-0-3-0-3$. By Lemma 5 a, we have $\sum_{v \in V(P)}\left(s^{\prime}(v)-0.8\right) \geq 1$, contradicting Equation (2).

Lemma 9. If $n \geq 5$ and $f$ is a $\gamma_{d R}$-function of $P(n, 2)$ with $f\left(v_{i}\right)=3$ for some $i \in[n]$, then $w(f) \geq\left\lceil\frac{8 n}{5}\right\rceil+1$.

Proof. Suppose to the contrary that there exists a $\gamma_{d R}$-function $f$ with $w(f)=\left\lceil\frac{8 n}{5}\right\rceil$ such that $f\left(v_{i}\right)=3$ for some $i \in[n]$. By Lemma 6, we have $f\left(u_{i}\right)=f\left(v_{i \pm 2}\right)=0$. Let $S=\left\{x \mid x \in N_{2}(v), f(x) \geq 2\right\}$. By Lemma 7, we have $1 \leq|S| \leq 2$, and we just need to consider the following two cases. 
Case 1: $|S|=1$.

We may without loss of generality assume that $\left\{f\left(u_{i-1}\right), f\left(u_{i-2}\right), f\left(v_{i-4}\right)\right\}=\{0,0,2\}$ or $\{0,0,3\}$ and $f\left(u_{i+1}\right)=f\left(u_{i+2}\right)=f\left(v_{i+4}\right)=0$. Since $u_{i+1}$ and $u_{i+2}$ need to be double Roman dominated, we have $f\left(v_{i+1}\right)=f\left(u_{i+3}\right)=3$, contradicting Lemma 8 .

Case 2: $|S|=2$.

Now, it is sufficient to consider the following two cases.

Case 2.1: $S \subseteq\left\{u_{i-1}, u_{i-2}, v_{i-4}\right\}$ and $f\left(u_{i+1}\right)=f\left(u_{i+2}\right)=f\left(v_{i+4}\right)=0$.

Since $u_{i+1}, u_{i+2}$ need to be double roman dominated, we have $f\left(v_{i+1}\right)=f\left(u_{i+3}\right)=3$, contradicting Lemma 8.

Case 2.2: $S=\left\{s_{1}, s_{2}\right\}$, where $s_{1} \in\left\{u_{i-1}, u_{i-2}, v_{i-4}\right\}$ and $s_{2} \in\left\{u_{i+1}, u_{i+2}, v_{i+4}\right\}$.

By Lemma $8, f\left(u_{k}\right) \neq 3$ for each $k \in\{1,2, \cdots, n\}$, and thus, $\left\{f\left(u_{i+1}\right), f\left(u_{i+2}\right), f\left(u_{i-2}\right)\right.$, $\left.f\left(u_{i-1}\right)\right\}=\{0,2\}$.

Then, we have $f\left(v_{i+4}\right)=f\left(v_{i-4}\right)=0$. Otherwise, $f\left(v_{i+4}\right) \neq 0$ or $f\left(v_{i-4}\right) \neq 0$. By symmetry, we may assume without loss of generality that $f\left(v_{i+4}\right) \neq 0$. Thus, we have $f\left(u_{i+1}\right)=f\left(u_{i+2}\right)=0$. Since $u_{i+1}, u_{i+2}$ need to be double Roman dominated, we have $f\left(v_{i+1}\right)=f\left(u_{i+3}\right)=3$, contradicting Lemma 8.

Now, it is sufficient to consider the following three cases.

Case 2.2.1: $f\left(u_{i+1}\right)=f\left(u_{i-1}\right)=2$.

By Lemma 6, we have $f\left(u_{i \pm 2}\right)=f\left(v_{i \pm 1}\right)=0$.

Since $u_{i+2}$ needs to be double Roman dominated and by Lemma 8 , we have $f\left(u_{i+3}\right)=2$. Since $v_{i+1}$ needs to be double Roman dominated, we have $f\left(v_{i+3}\right) \geq 2$. Thus, there exists an edge $e \in E_{\left\{2,2^{+}\right\}}^{f}$ a contradiction with Lemma 6.

Case 2.2.2: $f\left(u_{i+2}\right)=f\left(u_{i-2}\right)=2$.

By Lemma 6, we have $f\left(u_{i \pm 3}\right)=f\left(u_{i \pm 1}\right)=0$.

Since $u_{i+1}, u_{i-1}$ need to be double Roman dominated, we have $f\left(v_{i \pm 1}\right)=2$. Thus, there exists an edge $e \in E_{\{2,2\}}^{f}$, a contradiction with Lemma 6.

Case 2.2.3: $f\left(u_{i+1}\right)=f\left(u_{i-2}\right)=2$.

By Lemma 6, we have $f\left(u_{i-3}\right)=f\left(v_{i+1}\right)=f\left(u_{i+2}\right)=0$.

Since $u_{i+2}$ needs to be double Roman dominated, we have $f\left(u_{i+3}\right)=2$. By Lemma 6 , we have $f\left(v_{i+3}\right)=f\left(u_{i+4}\right)=0$. Since $u_{i+4}$ needs to be double Roman dominated and by Lemma 8 , we have $f\left(u_{i+5}\right)=2$. Since $v_{i+3}$ needs to be double Roman dominated, we have $f\left(v_{i+5}\right) \geq 2$. Thus, there exists an edge $e \in E_{\left\{2,2^{+}\right\}}^{f}$, a contradiction with Lemma 6 .

Lemma 10. Let $n \geq 5$ and $n \not \equiv 0(\bmod 5)$. If $f$ is a $\gamma_{d R}$-function of $P(n, 2)$, then $w(f) \geq\left\lceil\frac{8 n}{5}\right\rceil+1$.

Proof. Suppose to the contrary that $w(f)=\left\lceil\frac{8 n}{5}\right\rceil$. By Lemmas 8 and 9, we have $\left|V_{3}\right|=0$. Now, we have:

Claim 1. $\left|V_{2} \cap N(v)\right|=2$ for any $v \in V(P(n, 2))$ with $f(v)=0$.

Proof. Suppose to the contrary that there exists a vertex $v \in V(P(n, 2))$ with $f(v)=0$ and $\mid V_{2} \cap$ $N(v) \mid=3$. We consider the following two cases.

Case 1: $v=u_{i}$ for some $i \in[n]$. 
Since $\left|V_{2} \cap N(v)\right|=3$, we have $f\left(u_{i-1}\right)=f\left(u_{i+1}\right)=f\left(v_{i}\right)=2$. By Lemma 6, we have $f\left(u_{i \pm 2}\right)=$ $0, f\left(v_{i \pm 1}\right)=0$ and $f\left(v_{i \pm 2}\right)=0$. Since $v_{i+1}$ needs to be double Roman dominated, we have $f\left(v_{i+3}\right)=2$. Since $u_{i+2}$ needs to be double Roman dominated, we have $f\left(u_{i+3}\right)=2$. Since $v_{i+3} u_{i+3} \in E_{\{2,2\}}^{f}$ contradicting Lemma 6.

Case 2: $v=v_{i}$ for some $i \in[n]$.

Since $\left|V_{2} \cap N(v)\right|=3$, we have $f\left(v_{i-2}\right)=f\left(v_{i+2}\right)=f\left(u_{i}\right)=2$. By Lemma 6, we have $f\left(u_{i \pm 1}\right)=$ $f\left(u_{i \pm 2}\right)=f\left(v_{i \pm 4}\right)=0$. Since $u_{i+1}$ needs to be double Roman dominated, we have $f\left(v_{i+1}\right)=2$. Since $u_{i-1}$ needs to be double Roman dominated, we have $f\left(v_{i-1}\right)=2$. Since $v_{i+1} v_{i-1} \in E_{\{2,2\}}^{f}$ contradicting Lemma 6.

We assume without loss of generality that $f\left(u_{i}\right)=2$. By Lemma 6, we have $f\left(u_{i-1}\right)=0, f\left(v_{i}\right)=0$ and $f\left(u_{i+1}\right)=0$. Since $v_{i}$ needs to be double Roman dominated, we assume without loss of generality that $f\left(v_{i-2}\right)=2$. By Claim 1, we have $f\left(v_{i+2}\right)=0$. Since $f\left(v_{i-2}\right)=2$, together with Lemma 6, we have $f\left(u_{i-2}\right)=0$. Since $u_{i-1}$ needs to be double Roman dominated, we have $f\left(v_{i-1}\right)=2$. Then, by Lemma 6 , we have $f\left(v_{i+1}\right)=0$. Since $v_{i+2}$ needs to be double Roman dominated, we have $f\left(u_{i+2}\right)=2$. That is to say, we have:

$$
f\left(\mathcal{B}_{i}\right)=f\left(\begin{array}{lllll}
u_{i-2} & u_{i-1} & u_{i} & u_{i+1} & u_{i+2} \\
v_{i-2} & v_{i-1} & v_{i} & v_{i+1} & v_{i+2}
\end{array}\right)=\left(\begin{array}{lllll}
0 & 0 & 2 & 0 & 2 \\
2 & 2 & 0 & 0 & 0
\end{array}\right) .
$$

By repeatedly applying Claim 1 and Lemma $6, f(x)$ can be determined for each $x \in \mathcal{B}_{i+5}$, and we have $f\left(\mathcal{B}_{i}\right)=f\left(\mathcal{B}_{i+5}\right)$. It is straightforward to see that $w(f)=\left\lceil\frac{8 n}{5}\right\rceil$ only if $n \equiv 0(\bmod 5)$, a contradiction.

\section{Conclusions}

By Lemma 1, Proposition 2 and Lemma 10, we have

Theorem 1. If $n \geq 5$, then:

$$
\gamma_{d R}(P(n, 2))= \begin{cases}\left\lceil\frac{8 n}{5}\right\rceil, & n \equiv 0(\bmod 5) \\ \left\lceil\frac{8 n}{5}\right\rceil+1, & n \equiv 1,2,3,4(\bmod 5)\end{cases}
$$

Remark 1. Beeler et al. [7] proposed the concept of the double Roman domination. They showed that $2 \gamma(G) \leq$ $\gamma_{d R}(G) \leq 3 \gamma(G)$. Moreover, they suggested to find double Roman graphs.

In [17], it was proven that:

Theorem 2. If $n \geq 5$, then $\gamma(P(n, 2))=\left\lceil\frac{3 n}{5}\right\rceil$.

Therefore, we have that $P(n, 2)$ is not double Roman for all $n \geq 5$.

In fact, there exist many double Roman graphs among Petersen graph $P(n, k)$. For example, in [12], it was shown that $P(n, 1)$ is a double Roman graph for any $n \not \equiv 2(\bmod 4)$. Therefore, it is interesting to find other Petersen graphs that are double Roman.

Author Contributions: Z.S. contributes for supervision, methodology, validation, project administration and formal analysing. H.J., P.W., Y.R., J.-B.L. contribute for resources, some computations and wrote the initial draft of the paper which were investigated and approved by Z.S. and J.-B.L. wrote the final draft.

Funding: This research work is supported by Key Supported Disciplines of Guizhou Province Computer Application Technology (No. QianXueWeiHeZi ZDXX[2016]20); Anhui Province Key Laboratory of Intelligent Building \& Building Energy Saving.

Conflicts of Interest: The authors declare no conflict of interest. 


\section{References}

1. Xu, G. 2-rainbow domination in generalized Petersen graphs $P(n, 3)$. Discret. Appl. Math. 2009, 157, 2570-2573. [CrossRef]

2. Garey, M.R.; Johnson, D.S. Computers and Intractability: A Guide to the Theory of NP-Completeness; W. H. Freeman and Co.: San Francisco, CA, USA, 1979.

3. Henning, M.A. A Characterization of Roman trees. Discuss. Math. Graph Theory 2002, 22, 325-334. [CrossRef]

4. Ahangar, H.A.; Amjadi, J.; Sheikholeslami, S.M.; Volkmann, L.; Zhao, Y. Signed Roman edge domination numbers in graphs. J. Comb. Optim. 2016, 31, 333-346. [CrossRef]

5. Ore, O. Theory of Graphs; American Mathematical Society: Providence, RI, USA, 1967.

6. Shao, Z.; Xu, J.; Sheikholeslami, S.M.; Wang, S. The domination complexity and related extremal values of large 3D torus. Complexity 2018, 3041426. [CrossRef]

7. Beeler, R.A.; Haynes, T.W.; Hedetniemi, S.T. Double Roman domination. Discret. Appl. Math. 2016, 211, $23-29$. [CrossRef]

8. Liu, C.H.; Chang, G.J. Roman domination on 2-connected graphs. SIAM J. Discret. Math. 2012, 26, $193-205$. [CrossRef]

9. Liu, C.H.; Chang, G.J. Upper bounds on Roman domination numbers of graphs. Discret. Math. 2012, 312, 1386-1391. [CrossRef]

10. Liu, C.H.; Chen, S.H.; Chang, G.J. Edge Roman domination on graphs. Graphs Comb. 2016, 32, 1731-1747.

11. Pushpam, P.R.; Mai, T.N. Roman domination in unicyclic graphs. J. Discret. Math. Sci. Cryptogr. 2012, 15, 237-257. [CrossRef]

12. Shao, Z.; Jiang, H.; Li, Z.; Wu, P.; Zerovnik, J.; Zhang, X. Discharging approach for double Roman domination in graphs. IEEE Access 2018, accepted for publication.

13. Shao, Z.; Klavžar, S.; Li, Z.; Wu, P.; Xu, J. On the signed Roman k-domination: Complexity and thin torus graphs. Discret. Appl. Math. 2017, 233, 175-186. [CrossRef]

14. Zhang, X.; Li, Z.; Jiang, H.; Shao, Z. Double Roman domination in trees. Inf. Process. Lett. 2018, 134, 31-34. [CrossRef]

15. Li, Z.; Shao, Z.; Lang, F.; Zhang, X.; Liu, J.B. Computational complexity of outer-Independent total and total Roman domination numbers in trees. IEEE Access 2018, 6, 35544-35550. [CrossRef]

16. Tong, C.; Lin, X.; Yang, Y.; Luo, M. 2-rainbow domination of generalized Petersen graphs $P(n, 2)$. Discret. Appl. Math. 2009, 157, 1932-1937. [CrossRef]

17. Ebrahimi, B.J.; Jahanbakht, N.; Mahmoodian, E.S. Vertex domination of generalized Petersen graphs. Discret. Math. 2009, 309, 4355-4361. [CrossRef]

(C) 2018 by the authors. Licensee MDPI, Basel, Switzerland. This article is an open access article distributed under the terms and conditions of the Creative Commons Attribution (CC BY) license (http://creativecommons.org/licenses/by/4.0/). 